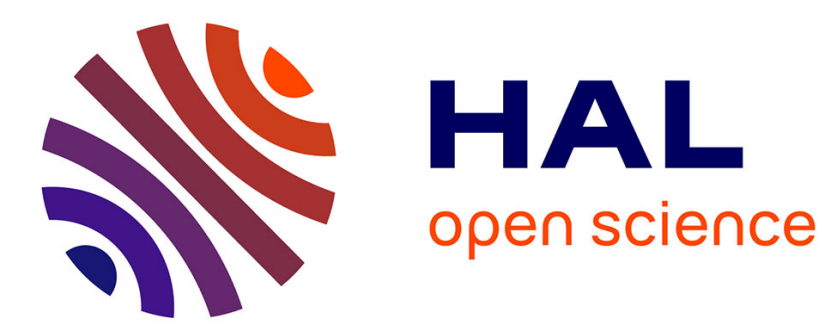

\title{
Optimization of characteristics of porous materials based on a modal synthesis method
}

Sébastien Besset, Louis Jezequel

\section{To cite this version:}

Sébastien Besset, Louis Jezequel. Optimization of characteristics of porous materials based on a modal synthesis method. European Journal of Mechanics - A/Solids, 2009, 28 (1), pp.102-109. 10.1016/j.euromechsol.2008.03.003 . hal-00625134

\section{HAL Id: hal-00625134 \\ https://hal.science/hal-00625134}

Submitted on 22 Sep 2011

HAL is a multi-disciplinary open access archive for the deposit and dissemination of scientific research documents, whether they are published or not. The documents may come from teaching and research institutions in France or abroad, or from public or private research centers.
L'archive ouverte pluridisciplinaire HAL, est destinée au dépôt et à la diffusion de documents scientifiques de niveau recherche, publiés ou non, émanant des établissements d'enseignement et de recherche français ou étrangers, des laboratoires publics ou privés. 


\title{
Optimization of characteristics of porous materials based on a modal synthesis method
}

\author{
S. Besset and L. Jézéquel \\ LTDS, Équipe D2S, UMR CNRS 5513 \\ École Centrale de Lyon \\ 36 av. Guy de Collongue \\ 69134 Ecully cedex, France \\ European Journal of Mechanics, A/Solids 28 (2009) 102-109

\begin{abstract}
Complex structures used in the automotive industry often include porous materials, in order to reduce the noise in acoustic cavities. The method proposed in this paper aims at optimizing the characteristics of these porous materials using modal criteria based on the modal analysis of the structure. The use of a generalize modal synthesis method allow to describe the structure and the cavities with sets of modes; the size of the resulting system is smaller, for only generalized degrees of freedom are used for each part of the coupled fluid-structure system. First, a modelization of the porous media is proposed, and the generalized modal analysis method is explained. An optimization is then processed on the thickness of the porous materials.
\end{abstract}

keyword: Modal analysis; Optimization; Porous media

\section{Introduction}

Acoustic problems in the automotive industry has long been studied, for the noise in acoustic cavities is a very important criterion for the customer. In order to reduce this noise, poroelastic materials are applied on the structure. In this paper, we optimize the thickness of these porous materials using optimization criteria based on a generalized modal analysis method.

The modal analysis method we use has been described in a previous paper [1] for a structural problem. It has then been extended to the case of a coupled fluid-structure problem [2]. The principle of this modal synthesis method is summarized in the paper. It is based on the modal description of each substructure and boundary using a "double" or "triple" modal synthesis, which have first been proposed by Jézéquel [3, 4].

It is possible to define optimization criteria based on the modal description of a coupled fluid-structure system. It has already been done in the case of a structural problem [5] and has been extended to the case of a coupled fluid-structure system [6]. 
Such criteria correspond to a systemic approach of vibroacoustic problems. The relation between the excitation and the pressure in the cavities can be expressed through modal matrices coming from the generalized modal analysis of the structure. Several terms appear in the expression of the pressure, which correspond to several "vibration paths". The expressions of these paths constitute the criteria we use for the optimization.

Porous materials have long been studied. Biot [7, 8, 9, 10] has first proposed a method to describe these materials. Later, Allard [11, 12] proposed a more precise modelization, introducing for example frequency dependant terms in the expression of the densities. Finite element models have also been proposed [13]. Our approach aims at describing porous materials through boundary impedances; some papers have already been written on the subject [14, 15], but the method we propose here uses these impedances to construct coupling terms of finite element matrices.

\section{Modal analysis of the structure}

The complex structure we consider in this paper include plates and two acoustic cavities. It has the same geometry of the structure considered in [5]. The geometry of the structure is shown in the figure 1 ;

- $\mathcal{F}_{1}$ is the first acoustic cavity, where the pressure level has to be minimized;

- $\mathcal{F}_{2}$ is the second acoustic cavity;

- $\mathcal{P}$ is the plate between the two cavities.

For figure 1 to be more understandable, the plates have not been drawn. The structure will be excited by a displacement applied on its skeleton.

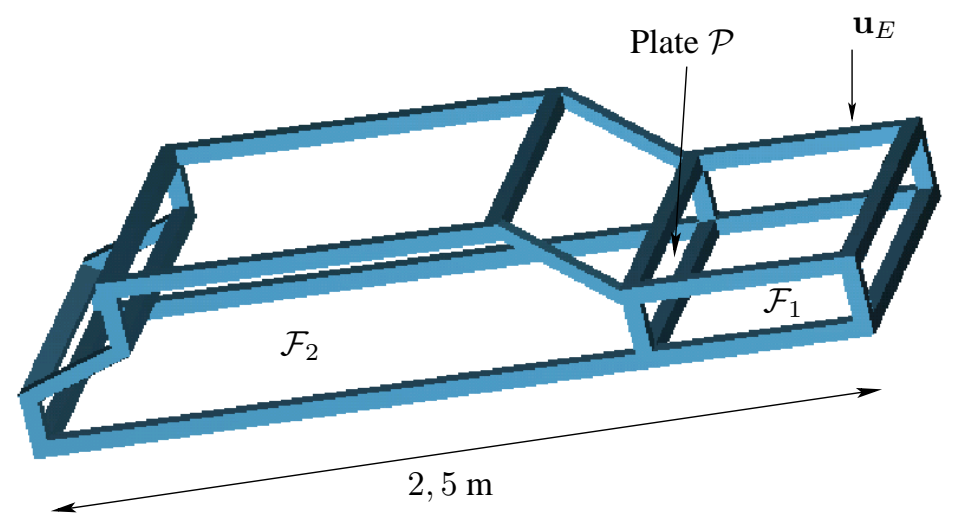

Figure 1: Structure to optimize

The motion equation of the coupled fluid-structure system is the classical equation, written in $(\mathbf{u}, \mathbf{p})$ formulation, without damping: 


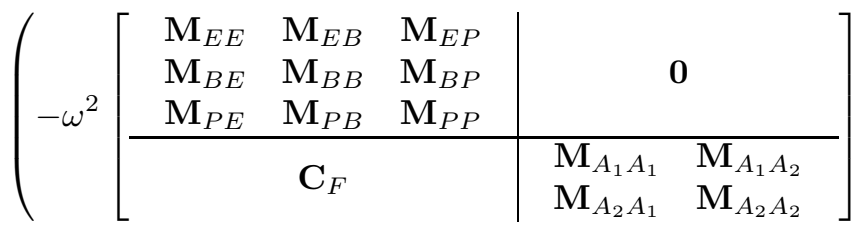

$$
\begin{aligned}
& \left.+\left[\begin{array}{ccc|cc}
\mathbf{K}_{E E} & \mathbf{K}_{E B} & \mathbf{K}_{E P} & \\
\mathbf{K}_{B E} & \mathbf{K}_{B B} & \mathbf{K}_{B P} & -\mathbf{C}_{F}^{T} \\
\mathbf{K}_{P E} & \mathbf{K}_{P B} & \mathbf{K}_{P P} & & \\
\hline & \mathbf{0} & & \mathbf{K}_{A_{1} A_{1}} & \mathbf{K}_{A_{1} A_{2}} \\
& \mathbf{K}_{A_{2} A_{1}} & \mathbf{K}_{A_{2} A_{2}}
\end{array}\right]\right)\left\{\begin{array}{c}
\mathbf{u}_{E} \\
\mathbf{u}_{B} \\
\mathbf{u}_{P} \\
\mathbf{p}_{1} \\
\mathbf{p}_{2}
\end{array}\right\}=\left\{\begin{array}{c}
\mathbf{f}_{E} \\
\mathbf{f}_{B} \\
\mathbf{f}_{P} \\
\mathbf{0} \\
\mathbf{0}
\end{array}\right\}
\end{aligned}
$$

$\mathbf{C}_{F}$ is a matrix coming from the fluid-structure coupling. In order to define the criteria for the optimization of the structure, we need to express this matrix more precisely:

$$
\mathbf{C}_{F}=\left[\begin{array}{lll}
\mathbf{M}_{A_{1} E} & \mathbf{M}_{A_{1} B} & \mathbf{M}_{A_{1} P} \\
\mathbf{M}_{A_{2} E} & \mathbf{M}_{A_{2} B} & \mathbf{M}_{A_{2} P}
\end{array}\right]=-\left[\begin{array}{ll}
\mathbf{K}_{E A_{1}} & \mathbf{K}_{E A_{2}} \\
\mathbf{K}_{B A_{1}} & \mathbf{K}_{B A_{2}} \\
\mathbf{K}_{P A_{1}} & \mathbf{K}_{P A_{2}}
\end{array}\right]^{T}
$$

Notice that $\mathbf{M}_{X_{i} Y_{j}}(\mathbf{i}=1,2$ and $\mathbf{j}=1,2)$ are not mass and stiffness terms. These terms are related to the discretized acoustic equation. That is why equation 2 can be written without units problems.

$\mathbf{u}_{B}, \mathbf{u}_{P}$ and $\mathbf{p}$ are the degrees of freedom of the system:

- $\mathbf{u}_{E}$ corresponds to the excited points of the structure;

- $\mathbf{u}_{B}$ corresponds to the boundaries between the plates;

- $\mathbf{u}_{P}$ corresponds to the plates;

- $\mathbf{p}_{1}$ are the degrees of freedom of the acoustical cavity $\mathcal{F}_{1}$;

- $\mathbf{p}_{2}$ are the degrees of freedom of the acoustical cavity $\mathcal{F}_{2}$.

The modal analysis method used to describe the system is based on the "double" and "triple" modal synthesis proposed in previous papers [1, 2, 3, 4]. Modes are used to describe the acoustic cavities, the boundaries and the plates. This method leads to the modal equation: 


$$
\begin{aligned}
& \left(\begin{array}{ccc|cc}
\overline{\mathbf{M}}_{E E} & \overline{\mathbf{M}}_{E B} & \overline{\mathbf{M}}_{E P} & & \\
\overline{\mathbf{M}}_{B E} & \overline{\mathbf{m}}_{B B} & \mathbf{0} & \mathbf{0} & \\
\overline{\mathbf{M}}_{P E} & \mathbf{0} & \overline{\mathbf{m}}_{P P} & & \\
\hline & \overline{\mathbf{C}}_{F} & & \overline{\mathbf{m}}_{A_{1} A_{1}} & \mathbf{0} \\
& & \mathbf{0} & \overline{\mathbf{m}}_{A_{2} A_{2}}
\end{array}\right]+i \omega\left[\begin{array}{ccccc}
\mathbf{c}_{E} & \mathbf{0} & \mathbf{0} & \mathbf{0} & \mathbf{0} \\
\mathbf{0} & \mathbf{c}_{B} & \mathbf{0} & \mathbf{0} & \mathbf{0} \\
\mathbf{0} & \mathbf{0} & \mathbf{c} & \mathbf{0} & \mathbf{0} \\
\mathbf{0} & \mathbf{0} & \mathbf{0} & \mathbf{0} & \mathbf{0} \\
\mathbf{0} & \mathbf{0} & \mathbf{0} & \mathbf{0} & \mathbf{0}
\end{array}\right] \\
& \left.+\left[\begin{array}{ccc|cc}
\overline{\mathbf{K}}_{E E} & \overline{\mathbf{K}}_{E B} & \overline{\mathbf{K}}_{E P} & -\overline{\mathbf{C}}_{F}^{T} \\
\overline{\mathbf{K}}_{B E} & \overline{\mathbf{k}}_{B B} & \mathbf{0} & - \\
\overline{\mathbf{K}}_{P E} & \mathbf{0} & \overline{\mathbf{k}}_{P P} & & \\
\hline & \mathbf{0} & & \overline{\mathbf{k}}_{A_{1} A_{1}} & \mathbf{0} \\
& & & \mathbf{0} & \overline{\mathbf{k}}_{A_{2} A_{2}}
\end{array}\right]\right)\left\{\begin{array}{c}
\mathbf{u}_{E} \\
\mathbf{q}_{B} \\
\mathbf{q}_{P} \\
\mathbf{q}_{A 1} \\
\mathbf{q}_{A 2}
\end{array}\right\}=\left\{\begin{array}{c}
\overline{\mathbf{f}}_{E} \\
\overline{\mathbf{f}}_{B} \\
\overline{\mathbf{f}}_{P} \\
\mathbf{0} \\
\mathbf{0}
\end{array}\right\}
\end{aligned}
$$

The modal damping matrix is assumed to be diagonal. The modal analysis described in equation 6 and 7 allows $\overline{\mathbf{k}}_{A_{i} A_{i}}, \overline{\mathbf{k}}_{P P}, \overline{\mathbf{k}}_{B B}, \overline{\mathbf{m}}_{P P}, \overline{\mathbf{m}}_{B B}$ and $\overline{\mathbf{m}}_{A_{i} A_{i}}(i=1$ or 2) to be diagonal matrices too. This equation will be used in section 4 to introduce optimization criteria. The modal matrices used for obtaining the generalized degrees of freedom are defined as follows:

$$
\begin{aligned}
& \mathbf{u}_{P}=\boldsymbol{\Phi}_{P} \mathbf{q}_{P}+\boldsymbol{\Psi}_{P B} \mathbf{u}_{B}+\boldsymbol{\Psi}_{P E} \mathbf{u}_{E} \\
& \mathbf{u}_{B}=\boldsymbol{\Phi}_{B} \mathbf{q}_{B}+\boldsymbol{\Psi}_{B E} \mathbf{u}_{E} \\
& \mathbf{p}_{1}=\boldsymbol{\Phi}_{A 1} \mathbf{q}_{A 1} \\
& \mathbf{p}_{2}=\boldsymbol{\Phi}_{A 2} \mathbf{q}_{A 2}
\end{aligned}
$$

Matrices $\boldsymbol{\Phi}_{P}, \boldsymbol{\Phi}_{B}, \boldsymbol{\Phi}_{A 1}$ and $\boldsymbol{\Phi}_{A 2}$ are the matrices of the modes of the structure:

- $\boldsymbol{\Phi}_{P}$ is the matrix corresponding to the mode of the plates when boundaries are fixed;

- $\boldsymbol{\Phi}_{B}$ is the matrix corresponding to the mode of the hollow parts constituting the skeleton of the structure, considering a static condensation of the plates on these hollow parts;

- $\boldsymbol{\Phi}_{A 1}$ is the matrix corresponding to the mode of the acoustical cavity $\mathcal{F}_{1}$ (cavity modes);

- $\boldsymbol{\Phi}_{A 2}$ is the matrix corresponding to the mode of the acoustical cavity $\mathcal{F}_{2}$ (cavity modes);

- $\boldsymbol{\Psi}_{P B}, \boldsymbol{\Psi}_{P E}$ and $\boldsymbol{\Psi}_{B E}$ are static modes of the structure.

\section{Analysis of the poroelastic materials}

The coupling terms recalled in equation 2 comes from the classical acoustical coupling equation: 


$$
\frac{\partial p}{\partial n}=\rho_{f} \omega^{2} \mathbf{u}_{n}
$$

where $\rho_{f}$ is the density of the fluid, $p$ is the pressure on the fluide-structure boundary, and $\mathbf{u}_{n}$ is the normal displacement. It is possible to introduce a boundary impedance $Z$ that allows to describe the behaviour of the boundary if we consider a poroelastic material on this boundary. In equation 8 is considered to be infinite. Taking into account the impedance $Z$, the coupling equation becomes:

$$
\frac{\partial p}{\partial n}-\frac{i \omega \rho_{f}}{Z} p=\rho_{f} \omega^{2} \mathbf{u}_{n}
$$

The aim of this section is to determinate $Z$ in function of the characteristics of a poroelastic material. The figure 2 shows the notations we use:

- $u_{0}$ is the displacement of the boundary between the porous material and the wall (a plate of the structure);

- $u_{l}$ is the displacement of the porous material at the boundary with the fluid cavity;

- $l$ is the thickness of the porous material.

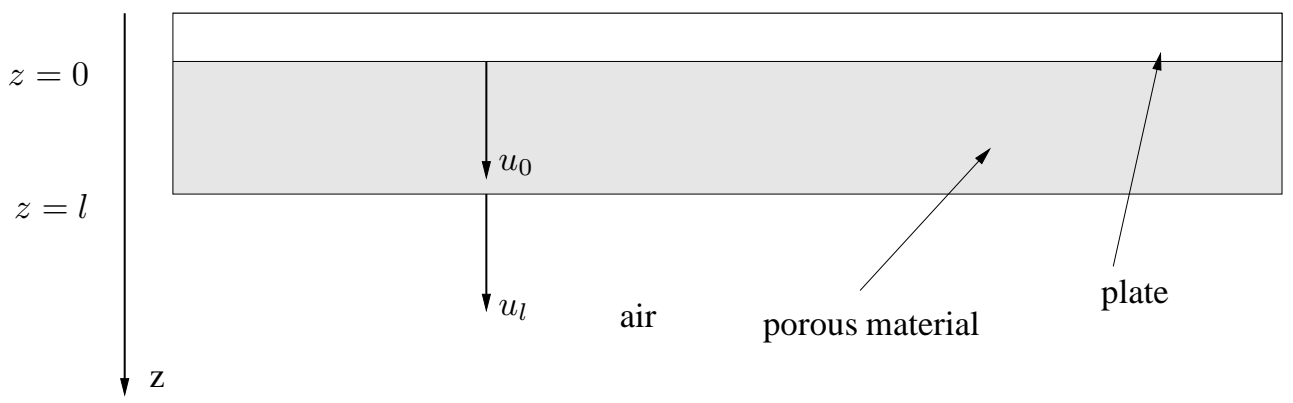

Figure 2: Notations on the purous material

At the boundary between an acoustic cavity and a porous material, we use the coupling equation proposed by Atalla [13]:

$$
\left(1-h-h \frac{Q}{R}\right) u_{l}-\left(\gamma u_{l}-\frac{h^{2}}{\tilde{\rho}_{22} \omega^{2}} \frac{\partial p}{\partial z}(z=l)\right)=\frac{1}{\tilde{\rho}_{0} \omega^{2}} \frac{\partial p}{\partial z}(z=l)
$$

In order to take into account the thickness of the porous material and the structure it is fixed on, we have to express $u_{l}$ in function of $u_{0}$. To do this, we consider the equation of the fluid part of the porous material [13], which is recalled here: 


$$
\Delta p+\frac{\tilde{\rho}_{22}}{R} \omega^{2} p-\frac{\tilde{\rho}_{22}}{h^{2}} \gamma \omega^{2} \operatorname{div} \mathbf{u}=0
$$

$h$ is the porosity of the porous material, $b$ is a cœefficient depending on the characteristics of the pores of the porous material and $\alpha_{\infty}$ is the tortuosity of the porous material. $\gamma, \tilde{\rho}_{22}$ and $\tilde{\rho}_{12}$ are defined as follows:

$$
\begin{gathered}
\tilde{\rho}_{22}=\rho_{22}-i \frac{b}{\omega} \\
\rho_{22}=h \rho_{f} \alpha_{\infty} \\
\gamma=h\left(\frac{\tilde{\rho}_{12}}{\tilde{\rho}_{22}}-\frac{Q}{R}\right) \\
\tilde{\rho}_{12}=\rho_{12}-i \frac{b}{\omega} \\
\rho_{12}=-h \rho_{f}\left(\alpha_{\infty}-1\right)
\end{gathered}
$$

$Q$ and $R$ are cœfficients that depend on the characteristics of the porous material. Their expression can be found in the book of Allard [11]. They take into account the thermal effects in the pore, so they have a frequency dependent complex amplitude that we recall:

$$
\begin{aligned}
& Q=(1-h) K_{f} \\
& R=h K_{f} \\
& K_{f}=\frac{\gamma P_{0}}{\gamma-(\gamma-1)\left(1+\frac{8 \eta}{i \Lambda^{2} B^{2} \omega \rho_{f}}\left(1+i \rho_{f} \omega B^{2} \frac{\Lambda^{2}}{16 \eta}\right)^{1 / 2}\right)^{-1}}
\end{aligned}
$$

where $B$ and $P_{0}$ depend on the temperature. For $18^{\circ}$, we have $B=\sqrt{0,71}$ and $P_{0}=1,0132.10^{5}$ (see [11]).

Several expressions do exist to evaluate $b$ which depends on the frequency. We choose to use an expression proposed in [11]:

$$
b=\sigma h^{2} \frac{G(\omega)}{\omega}
$$

where:

$$
G(\omega)=\sqrt{\frac{1+4 i \alpha_{\infty}^{2} \eta \rho_{f} \omega}{\sigma^{2} \Lambda^{2} h^{2}}}
$$

with the notations:

- $\Lambda$ is a characteristic dimension of the porous material;

- $\sigma$ is the flow resistivity (intrinsic property of the material); 
- $\eta$ is the viscosity;

Considering only the $z$ displacement, equation 11 can be written:

$$
\frac{\partial^{2} p}{\partial z^{2}}+\omega^{2} \frac{\tilde{\rho}_{22}}{R} p-\omega^{2} \frac{\tilde{\rho}_{22}}{h^{2}} \gamma \frac{\partial u}{\partial z}=0
$$

Considering that the thickness $l$ is small (for example $l<\frac{2 \pi c_{0}}{\omega}$ ), it is possible to write:

$$
\begin{aligned}
& \frac{\partial u}{\partial z}=\frac{u_{l}-u_{0}}{l} \\
& \frac{\partial^{2} p}{\partial z^{2}}=\frac{\frac{\partial p}{\partial z}(z=l)-\frac{\partial p}{\partial z}(z=0)}{l}
\end{aligned}
$$

If the boundary between the porous material and the structure is rigid, $\frac{\partial p}{\partial z}(z=0)$ can be expressed as follows:

$$
\frac{\partial p}{\partial z}(z=0)=\rho_{0} \omega^{2} u_{0}
$$

The equation 23 becomes then:

$$
\frac{1}{l} \frac{\partial p}{\partial z}(z=l)+\omega^{2} \frac{\tilde{\rho}_{22}}{R} p-\omega^{2} \frac{\tilde{\rho}_{22}}{h^{2} l} \gamma\left[u_{l}-\left(1-\frac{h^{2} \rho_{0}}{\tilde{\rho}_{22} \gamma}\right) u_{0}\right]=0
$$

This equation allows to express $u_{l}$ in function of $u_{0}$ :

$$
u_{l}=\left(1-\frac{h^{2} \rho_{0}}{\tilde{\rho}_{22} \gamma}\right) u_{0}+\frac{h^{2}}{\omega^{2} \tilde{\rho}_{22} \gamma} \frac{\partial p}{\partial z}+\frac{h^{2} l}{R \gamma} p
$$

The coupling equation becomes:

$$
\begin{aligned}
{\left[\frac{1}{\rho_{0}}-\left(1-h-h \frac{Q}{R}\right) \frac{h^{2}}{\tilde{\rho}_{22} \gamma}\right] } & \frac{\partial p}{\partial z}-\omega^{2}\left(1-h-h \frac{Q}{R}-\gamma\right) \frac{h^{2} l}{R \gamma} p \\
& =\omega^{2}\left(1-h-h \frac{Q}{R}-\gamma\right) \frac{h^{2} l}{R \gamma}\left(1-\frac{h^{2} \rho_{0}}{\tilde{\rho}_{22} \gamma}\right) u_{0}
\end{aligned}
$$

which is close to the classical equation of coupling:

$$
\frac{\partial p}{\partial z}-\frac{j \omega \rho_{0}}{Z_{p}} p=\rho_{0} \omega^{2} \xi_{2} u_{0}
$$

with the notations: 


$$
\begin{aligned}
Z_{p} & =j \frac{R \gamma}{\omega h^{2} l \xi_{1}} \\
\xi_{1} & =\frac{1-h-h \frac{Q}{R}-\gamma}{1-\rho_{0}\left(1-h-h \frac{Q}{R}\right) \frac{h^{2}}{\tilde{\rho}_{22} \gamma}} \\
\xi_{2} & =\frac{h^{2} l}{R \gamma} \frac{\left(1-h-h \frac{Q}{R}-\gamma\right)\left(1-\frac{h^{2} \rho_{0}}{\tilde{\rho}_{22}}\right)}{1-\rho_{0}\left(1-h-h \frac{Q}{R}\right) \frac{h^{2}}{\tilde{\rho}_{22} \gamma}}
\end{aligned}
$$

The modelization retained for the porous materials is a simplified modelization. It is possible to study the influence of the simplifications by comparing the impedance $Z_{p}$ we propose with the classical acoustic impedance, given by the relation:

$$
Z=\frac{p(l)}{i \omega u(l)}
$$

where $p(l)$ is the pressure and $i \omega u(l)$ is the speed of the fluid at $z=l$ (see figure2). The characteristics of the porous material used for the comparison between $Z_{p}$ and the impedance proposed by Allard [11] are recalled in table 1.

\begin{tabular}{|c|c|c|c|c|}
\hline $\begin{array}{c}\text { Tortuosity } \\
\alpha_{\infty}\end{array}$ & $\begin{array}{c}\text { Density } \\
\text { of the skeleton }\end{array}$ & $\begin{array}{c}\text { Porosity } \\
h\end{array}$ & $\begin{array}{c}\text { Poisson's } \\
\text { ratio }\end{array}$ & $\begin{array}{c}\text { Lamé Modulus } \\
N\end{array}$ \\
\hline \hline 1,06 & $130 \mathrm{kgm}^{-3}$ & 0,94 & 0 & $220(1+0,1 i)$ \\
\hline \hline \multicolumn{1}{|c|}{$\Lambda$} & $\sigma$ & \multicolumn{2}{|c|}{$\eta$} & $\rho_{f}$ \\
\hline \hline $1,1.10^{-4} \mathrm{~m}$ & $40000 \mathrm{Nm}^{-4} \mathrm{~s}$ & $1,84.10^{-5} \mathrm{Pl}$ & $1,213 \mathrm{kgm}^{-3}$ \\
\hline
\end{tabular}

Table 1: Characteristics of the porous material used in the comparison

The results of the comparison are given in the figures 3(a), 3(b), 4(a), 4(b), 5(a) and 5(b), corresponding to thicknesses of $1 \mathrm{~cm}, 5 \mathrm{~mm}$, and $2 \mathrm{~mm}$. The results are good if the thickness $l$ is small. Notice that the imaginary part of the impedance is always good, even if the thickness is not small. This imaginary part corresponds to the damping due to the porous material.

The equation 30 is a boundary condition between the fluid and the plates that generates coupling terms depending on the frequency, for the impedance $Z_{p}$ depends on the frequency. This coupling term can be expressed in the base of the modes of the structure defined in section 2, hence the equation:

$$
\left(-\omega^{2} \overline{\mathbf{M}}+i \omega \overline{\mathbf{C}}(\omega)+\overline{\mathbf{K}}\right) \overline{\mathbf{u}}=\overline{\mathbf{f}}
$$

where $\overline{\mathbf{C}}(\omega)$ is a complex matrix, depending on $\omega$. This matrix corresponds to the impedance $Z_{p}$ defined in equation $31, \overline{\mathbf{M}}, \overline{\mathbf{K}}, \overline{\mathbf{u}}$ and $\overline{\mathbf{f}}$ correspond to the matrices and vectors defined in equation 3 Using the poroelastic modelization we propose, equation 3 becomes: 


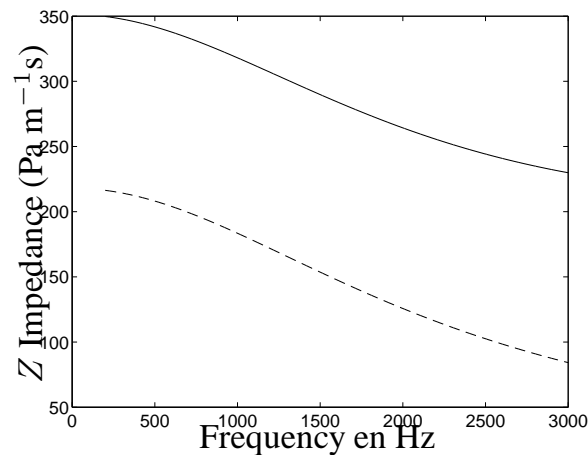

(a) Real part

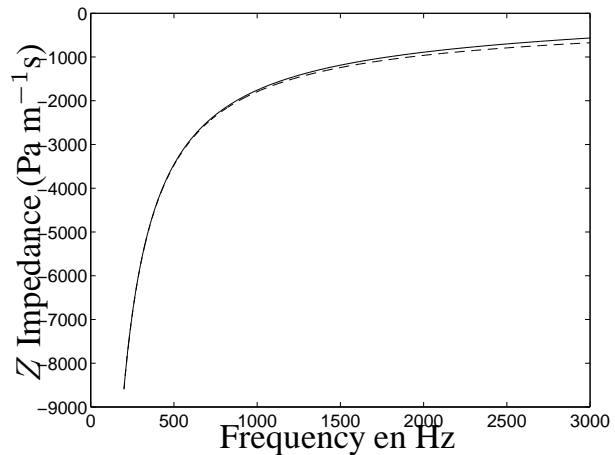

(b) Imaginary part

Figure 3: Boundary impedance for a porous material - thickness: $1 \mathrm{~cm}(-$ : Reference, - - : Proposed model $Z$ )

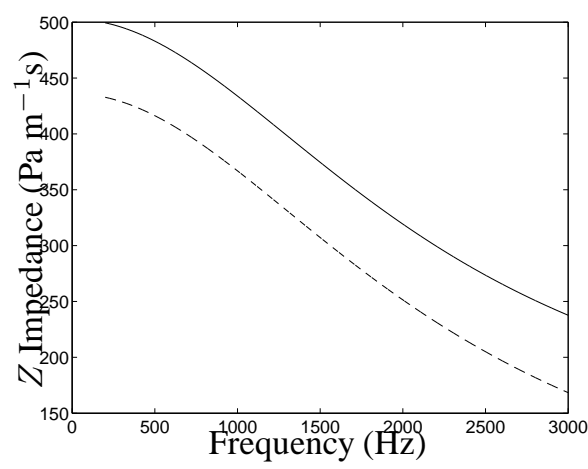

(a) Real part

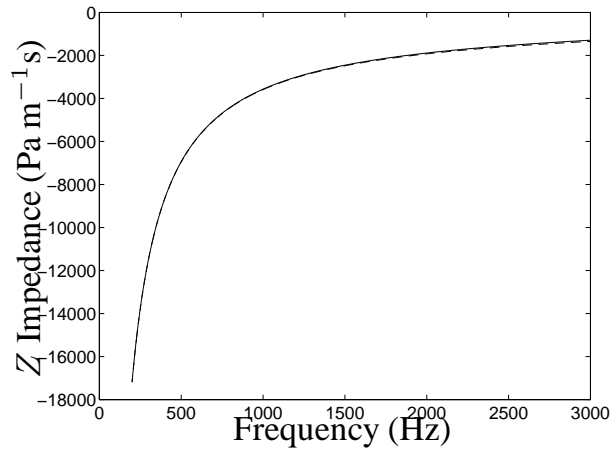

(b) Imaginary part

Figure 4: Boundary impedance for a porous material - thickness: $5 \mathrm{~mm}(-$ : Reference, - - : Proposed model $Z$ )

$$
\begin{aligned}
& \left(\begin{array}{ccc|cc}
\overline{\mathbf{M}}_{E E} & \overline{\mathbf{M}}_{E B} & \overline{\mathbf{M}}_{E P} & & \\
\overline{\mathbf{M}}_{B E} & \overline{\mathbf{m}}_{B B} & \mathbf{0} & \mathbf{0} & \\
\overline{\mathbf{M}}_{P E} & \mathbf{0} & \overline{\mathbf{m}}_{P P} & & \\
\hline & \overline{\mathbf{C}}_{F}(\omega) & & \overline{\mathbf{m}}_{A_{1}} & \mathbf{0} \\
& & \mathbf{0} & \overline{\mathbf{m}}_{A_{2}}
\end{array}\right]+i \omega\left[\begin{array}{ccc|cc}
\mathbf{c}_{E} & \mathbf{0} & \mathbf{0} & & \\
\mathbf{0} & \mathbf{c}_{B} & \mathbf{0} & \mathbf{0} & \\
\mathbf{0} & \mathbf{0} & \mathbf{c}_{P} & & \\
\hline & \mathbf{0} & & \mathbf{c}_{A 1}(\omega) & \mathbf{0} \\
& & \mathbf{0} & \mathbf{c}_{A 2}(\omega)
\end{array}\right]
\end{aligned}
$$

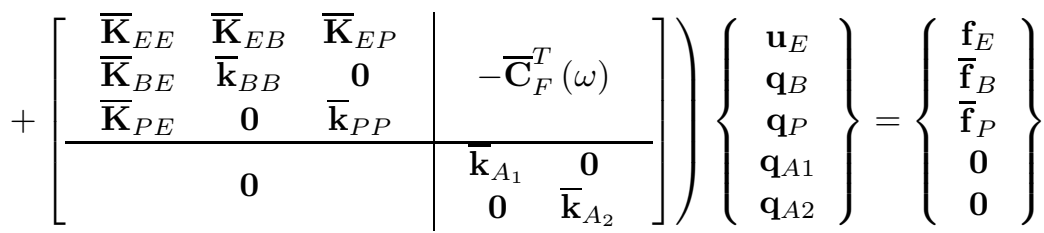




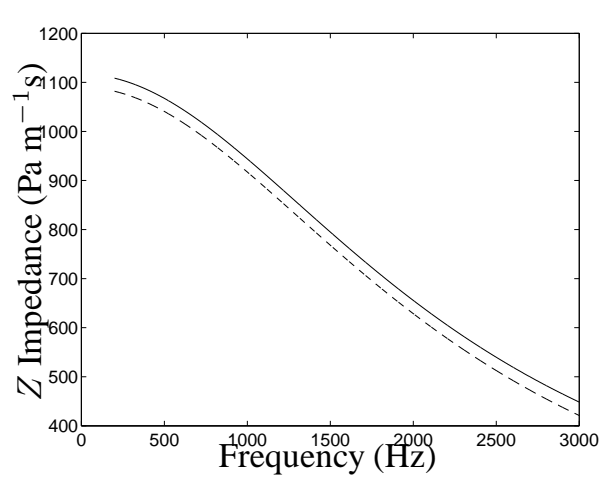

(a) Real part

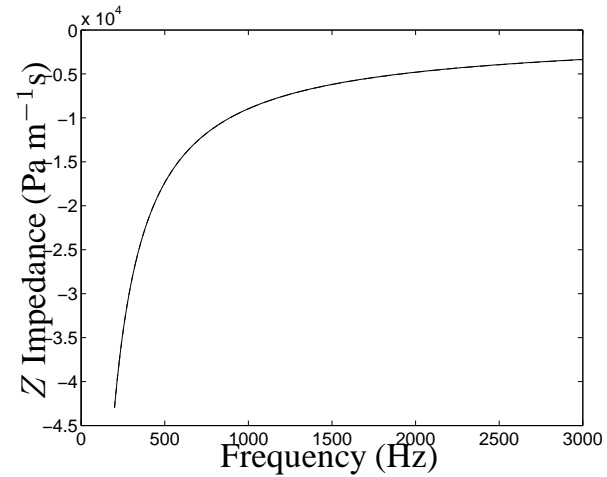

(b) Imaginary part

Figure 5: Boundary impedance for a porous material - thickness: $2 \mathrm{~mm}(-$ : Reference, - - : Proposed model $Z$ )

where the $\mathbf{q}_{i}$ are generalized degrees of freedom, whereas $\mathbf{u}_{E}$ are nodal degrees of freedom. The modal analysis is proceeded as proposed in section 2 - ie. without the porous material. The motion equation of the structure including porous materials is then written by projection on the modes calculated without it. $\mathbf{c}_{A 1}(\omega)$ and $\mathbf{c}_{A 2}(\omega)$ are assumed to be diagonal (Basile's hypothesis).

\section{Optimization criteria}

The optimization criteria we propose in this paper are based on the relationship between the pressure in the cavities and an excitation in displacement on the structure. The excited degrees of freedom are denoted $\mathbf{u}_{E}$. We are interested in the pressure level in the second cavity $\mathcal{F}_{2}$. The last line of equation 36 allows to express the pressure $\mathbf{p}_{2}$ in the second cavity $\mathcal{F}_{2}$. First, it is necessary to express $\mathbf{q}_{A 2}$ :

$$
\mathbf{q}_{A 2}=\frac{\omega^{2}\left(\overline{\mathbf{M}}_{A_{2} E}^{k} \mathbf{u}_{E}+\overline{\mathbf{M}}_{A_{2} B}^{k} \mathbf{q}_{B}+\overline{\mathbf{M}}_{A_{2} P}^{k} \mathbf{q}_{P}\right)}{-\omega^{2} \overline{\mathbf{m}}_{A 2}^{k}+\overline{\mathbf{k}}_{A_{2}}^{k}+i \omega \mathbf{c}_{A_{2}}^{k}(\omega)}
$$

We denote $\boldsymbol{\Phi}_{A_{2}}$ the matrix of the modes of the second cavity. $\boldsymbol{\Phi}_{A_{2}}^{k}$ is the $k^{\text {th }}$ column of $\boldsymbol{\Phi}_{A_{2}}$. The pressure $\mathbf{p}_{2}$ in $\mathcal{F}_{2}$ can then be written:

$$
\mathbf{p}_{2}=\boldsymbol{\Phi}_{A_{2}} \mathbf{q}_{A 2}=\sum_{k} \boldsymbol{\Phi}_{A_{2}}^{k} \mathbf{q}_{A 2}
$$

Replacing $\mathbf{q}_{A 2}$ in equation 38 with the expression given in equation 37 we obtain the relationship: 


$$
\begin{aligned}
\mathbf{p}_{2}= & \sum_{k}\left[\frac{\omega^{2} \boldsymbol{\Phi}_{A_{2}}^{k} \overline{\mathbf{M}}_{A_{2} E}^{k}}{-\omega^{2} \overline{\mathbf{m}}_{A 2}^{k}+\overline{\mathbf{k}}_{A_{2}}^{k}+i \omega \mathbf{c}_{A_{2}}^{k}(\omega)}\right] \mathbf{u}_{E} \\
& +\sum_{k}\left[\frac{\omega^{2} \boldsymbol{\Phi}_{A_{2}}^{k} \overline{\mathbf{M}}_{A_{2} B}^{k}}{-\omega^{2} \overline{\mathbf{m}}_{A 2}^{k}+\overline{\mathbf{k}}_{A_{2}}^{k}+i \omega \mathbf{c}_{A_{2}}^{k}(\omega)}\right] \mathbf{q}_{B} \\
& +\sum_{k}\left[\frac{\omega^{2} \mathbf{\Phi}_{A_{2}}^{k} \overline{\mathbf{M}}_{A_{2} P}^{k}}{-\omega^{2} \overline{\mathbf{m}}_{A 2}^{k}+\overline{\mathbf{k}}_{A_{2}}^{k}+i \omega \mathbf{c}_{A_{2}}^{k}(\omega)}\right] \mathbf{q}_{P}
\end{aligned}
$$

$\mathbf{q}_{B m}$ and $\mathbf{q}_{P}$ are now expressed in function of the nodal degrees of freedom, thanks to the relations coming from the modal analysis of the system and defined in equations 4, 5, 6 and7

$$
\begin{aligned}
& \mathbf{q}_{B}=\tilde{\boldsymbol{\Phi}}_{B}\left(\mathbf{u}_{B}-\boldsymbol{\Psi}_{B E} \mathbf{u}_{E}\right) \\
& \mathbf{q}_{P}=\tilde{\boldsymbol{\Phi}}_{P}\left(\mathbf{u}_{P}-\boldsymbol{\Psi}_{P B} \mathbf{u}_{B}-\boldsymbol{\Psi}_{P E} \mathbf{u}_{E}\right)
\end{aligned}
$$

where $\tilde{\boldsymbol{\Phi}}_{B}$ and $\tilde{\boldsymbol{\Phi}}_{P}$ are defined as follows:

$$
\begin{aligned}
\tilde{\mathbf{\Phi}}_{B} & =\boldsymbol{\Phi}_{B}^{T} \tilde{\mathbf{M}}_{B} \\
\tilde{\mathbf{\Phi}}_{P} & =\boldsymbol{\Phi}_{P}^{T} \tilde{\mathbf{M}}_{P}
\end{aligned}
$$

which can be written thanks to the eigenvectors property:

$$
\begin{aligned}
& \boldsymbol{\Phi}_{B}^{T} \tilde{\mathbf{M}}_{B} \boldsymbol{\Phi}_{B}=\mathbf{I} \\
& \boldsymbol{\Phi}_{P}^{T} \tilde{\mathbf{M}}_{P} \boldsymbol{\Phi}_{P}=\mathbf{I}
\end{aligned}
$$

where $\tilde{\mathbf{M}}_{B}$ is the condensed mass matrix used to find $\boldsymbol{\Phi}_{B}$, and $\tilde{\mathbf{M}}_{P}$ is the condensed mass matrix used to find $\boldsymbol{\Phi}_{P}$.

We can now define a criterion $\tilde{C}_{d}$ linked to the direct path between the displacement $\mathbf{u}_{E}$ and the pressure $\mathbf{p}_{2}$ :

$$
\tilde{C}_{d}=\sum_{k} \frac{\omega^{2} \boldsymbol{\Phi}_{A_{2}}^{k}\left(\overline{\mathbf{M}}_{A_{2} E}^{k}-\overline{\mathbf{M}}_{A_{2} B}^{k} \tilde{\boldsymbol{\Phi}}_{B} \boldsymbol{\Psi}_{B E}-\overline{\mathbf{M}}_{A_{2} P}^{k} \tilde{\boldsymbol{\Phi}}_{P} \boldsymbol{\Psi}_{P E}\right)}{-\omega^{2} \overline{\mathbf{m}}_{A 2}^{k}+\overline{\mathbf{k}}_{A_{2}}^{k}+i \omega \mathbf{c}_{A_{2}}^{k}(\omega)}
$$

In order to reduce the pressure level for the maxima, we consider criterion $\tilde{C}_{d}$ :

$$
C_{d}=\max _{k}\left|\frac{\omega_{k} \boldsymbol{\Phi}_{A_{2}}^{k}\left(\overline{\mathbf{M}}_{A_{2} E}^{k}-\overline{\mathbf{M}}_{A_{2} B}^{k} \tilde{\boldsymbol{\Phi}}_{B} \boldsymbol{\Psi}_{B E}-\overline{\mathbf{M}}_{A_{2} P}^{k} \tilde{\boldsymbol{\Phi}}_{P} \boldsymbol{\Psi}_{P E}\right)}{i \mathbf{c}_{A_{2}}^{k}\left(\omega_{k}\right)}\right|
$$


It is possible to define other criteria linked to other paths, as shown on figure 6 but this is not the purpose of the paper. We will only deal with the criterion $C_{d}$ to optimize the thickness of the porous media of the structure, for it is the main criterion, directly linked to the direct path between the pressure field and the excitation point (black arrow on figure 6).

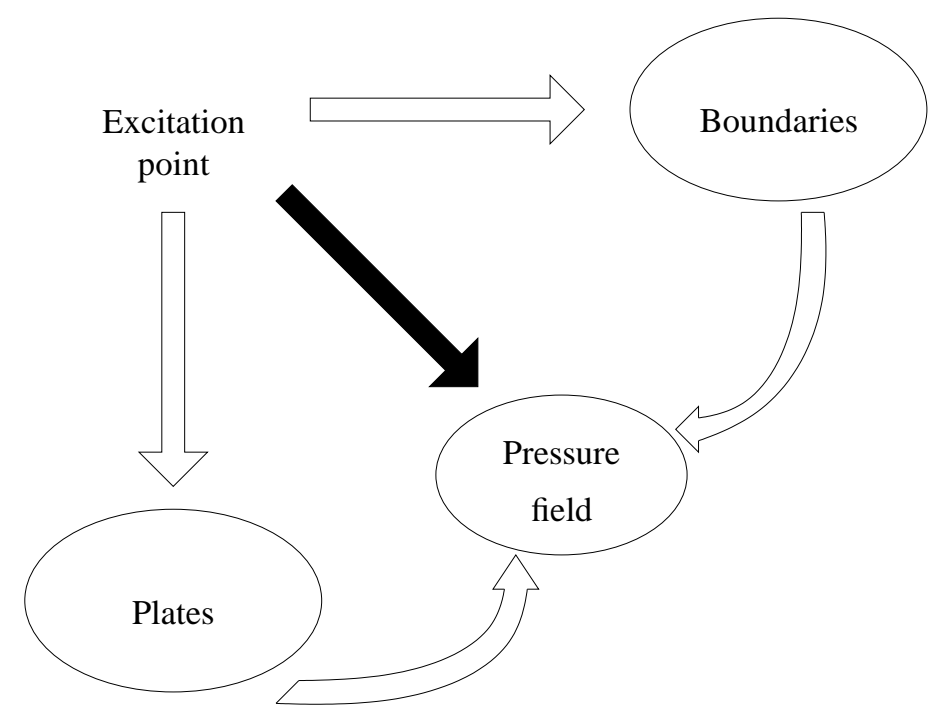

Figure 6: Direct path (in black) taken into account for optimization

\section{Optimization of the structure}

The optimization we use in this paper is based on two criteria. Criterion $C_{d}$ has been defined in section 4. Criterion $C_{m}$ is linked to the mass of the poroelastic material, which must not be too high. Four parts of porous materials are considered here:

- porous material 1: foam put on the plates around the second cavity $\mathcal{F}_{2}$;

- porous material 2: foam put on the plate $\mathcal{P}$ situated between the two cavities, on the side situated in the second cavity;

- porous material 3: foam put on the plate $\mathcal{P}$ situated between the two cavities, on the side situated in the first cavity;

- porous material 4: foam put on the plates around the first cavity $\mathcal{F}_{1}$;

The optimization algorithm considered here is a classical genetic algorithm that is given on figure 7

The results of the optimization are given on the Pareto diagram of the figure 8 , This diagram shows the optimal points: some of these points are better according to 


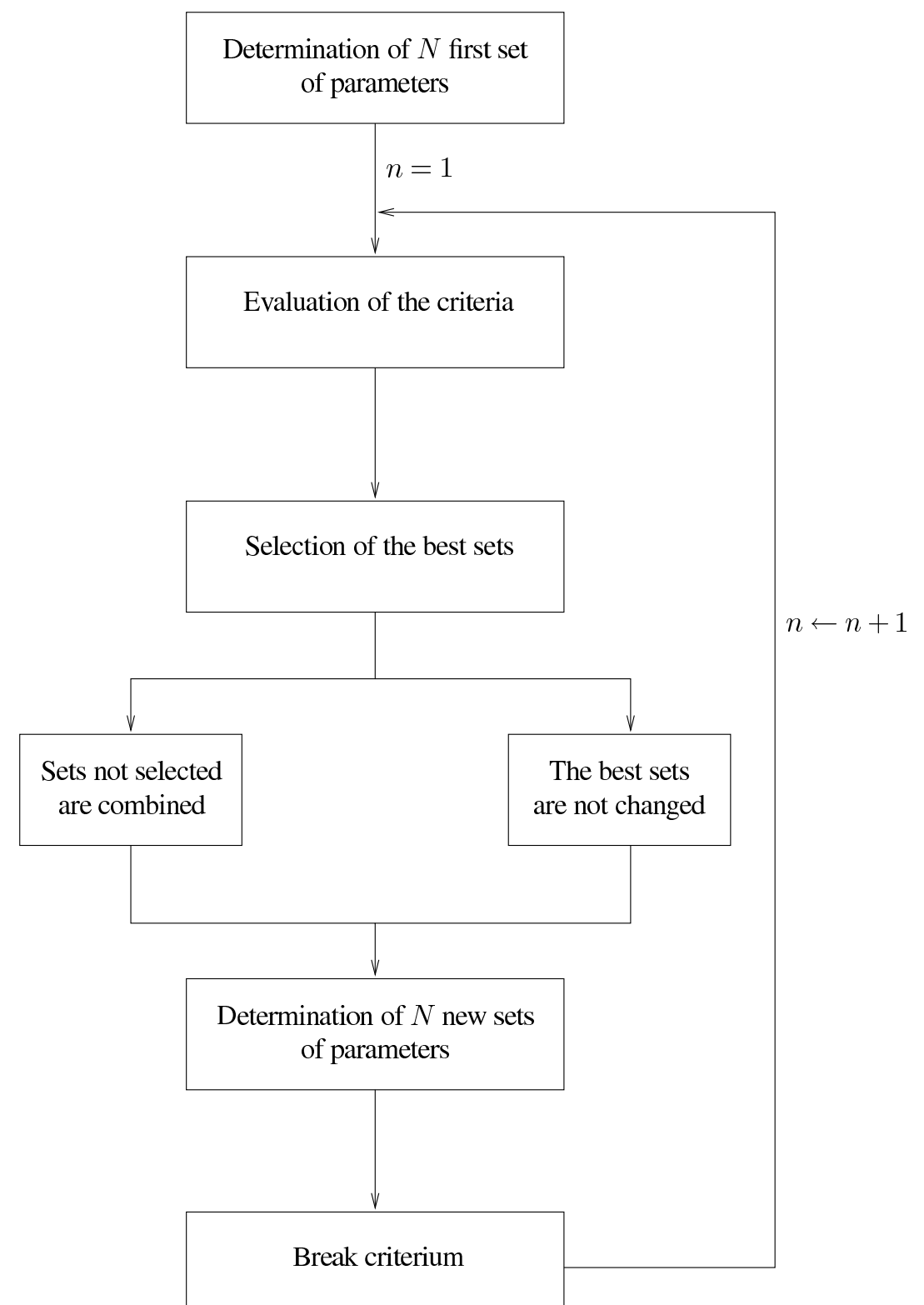

Figure 7: Genetic algorithm used for optimization

$C_{d}$, others are better according to $C_{m}$. Figures $9,10,11$ and 12 show the variation of the thicknesses of the foams in function of the mass of the structure - various optimized points are represented in these figures. We observe that the thicknesses of the foams corresponding to the first cavity are first increased. Figure 13 shows the pressure level in the cavity for the different steps of the optimization. Notice that other paths should 
be taken into account to get better results. Nevertheless, multicriteria optimization is not the purpose of the paper.

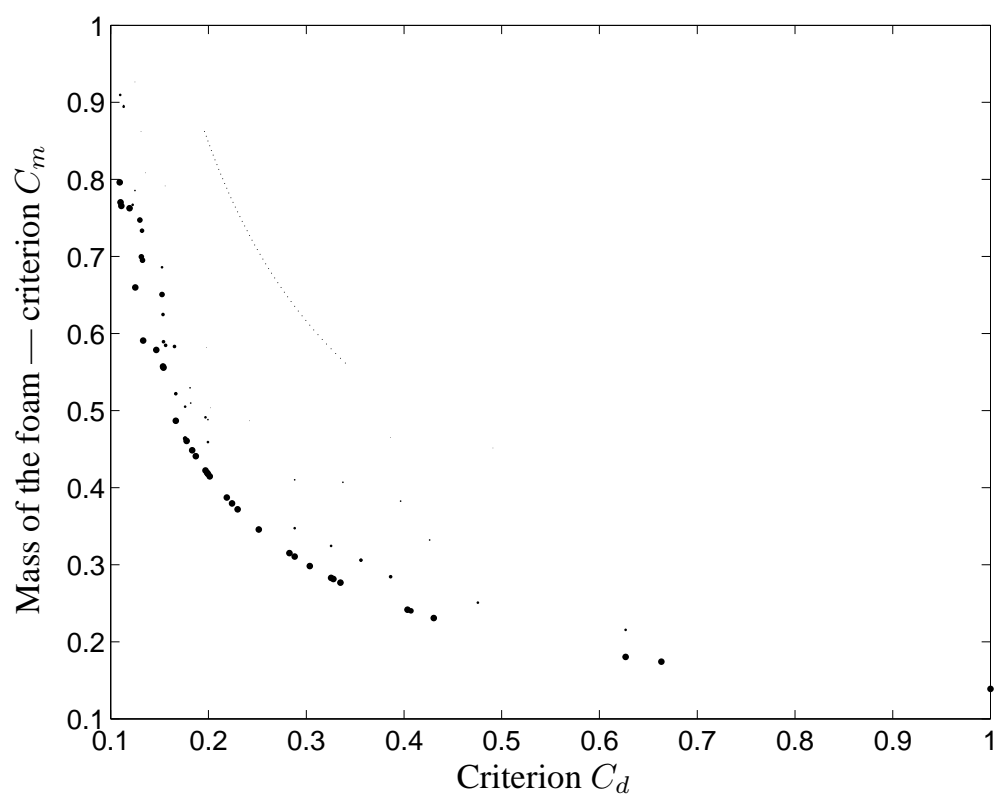

Figure 8: Pareto diagram of the optimization

\section{Conclusion}

A modelization of porous materials has been proposed, in order to be able to study the dynamic behaviour of a complex structure. A vibroacoustic criterion has been proposed, which allows to optimize the thicknesses of the porous materials used in the modeling of the structure. A genetic optimization has been processed, and we observe that the thicknesses of the foams corresponding to the first cavity are first increased.

The method allows to optimize complex structures including poroelastic components:

- The modes of the structure are first computed;

- Porous media are then analyzed through a frequency dependent and complex impedance;

- Generalized degrees of freedom of the structure are expressed in function of the modes of the structure; 


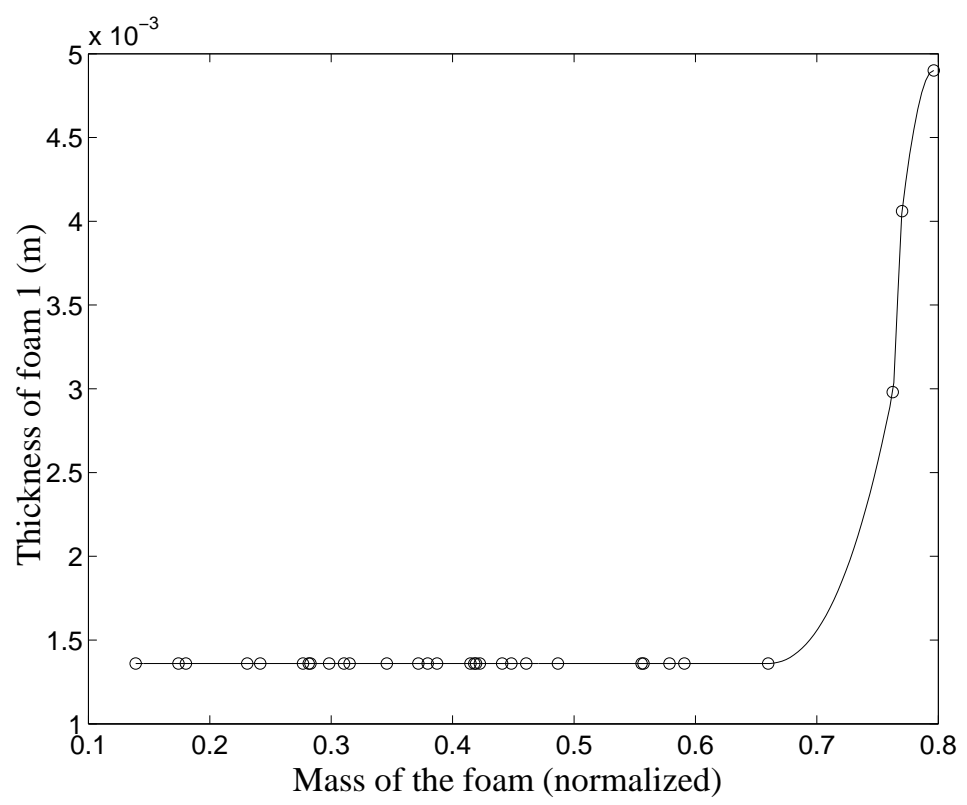

Figure 9: Best thickness of foam 1 in function of the mass of the foam

- The optimization is processed.

Notice that the example given in the paper show how to use the method on a given structure. Nevertheless, other structures may be considered, for the method can easily be generalized to other situations.

\section{References}

[1] S. Besset, L. Jézéquel, Dynamic substructuring based on a double modal analysis, Journal of Vibration and Acoustics (in press).

[2] S. Besset, L. Jézéquel, Triple modal synthesis for complex structures analysis, in: RASD2006, Southampton, UK, 2006.

[3] L. Jézéquel, H. D. Setio, Component modal synthesis methods based on hybrid models, part i : Theory of hybrid models and modal truncation methods, ASME Journal of Applied Mechanics 61 (1994) 100-108.

[4] L. Jézéquel, H. D. Setio, Component modal synthesis methods based on hybrid models, part ii : Numerical tests and experimental identification of hybrid model, ASME Journal of Applied Mechanics 61 (1994) 109-116. 


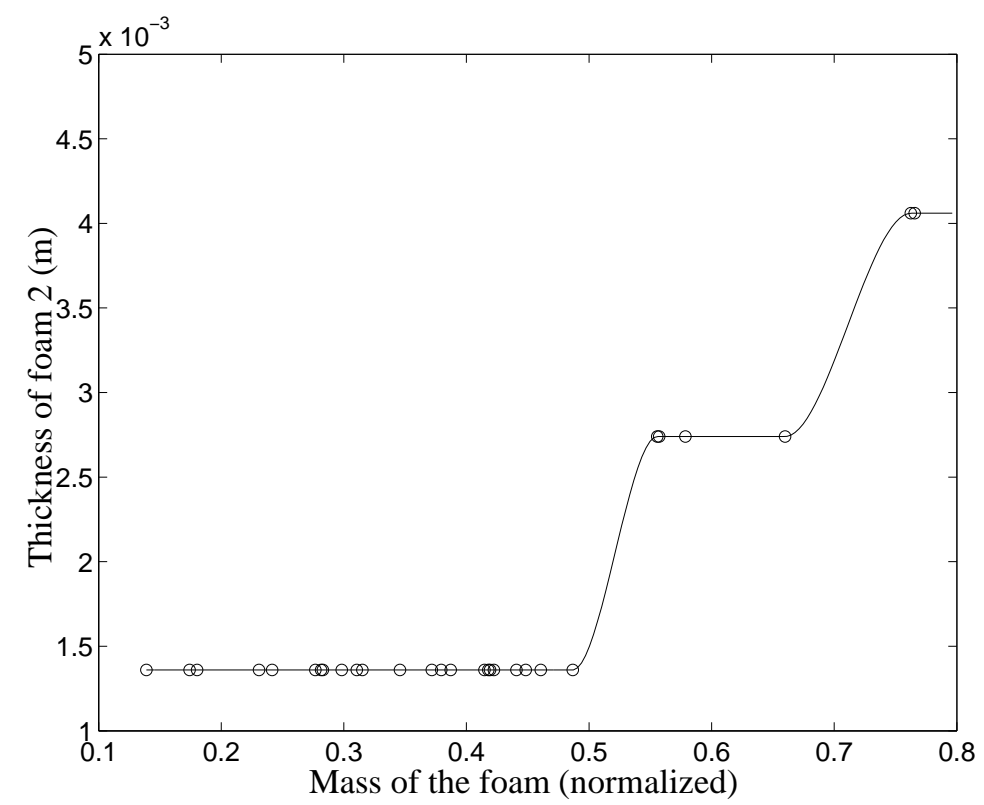

Figure 10: Best thickness of foam 2 in function of the mass of the foam

[5] S. Besset, L. Jézéquel, Optimization of structural dynamic behaviour based on effective modal parameters, International Journal for Numerical Methods in Engineering (in press).

[6] S. Besset, L. Jézéquel, Optimization of a coupled fluid-structure system using a modal approach, in: ICSV13, Vienna, Austria, 2006.

[7] M. Biot, Theory of propagation of elastic waves in a fluid-filled-saturated porous solid, Journal of the Acoustical Society of America 28 (1956) 168-178.

[8] M. Biot, Theory of propagation of elastic waves in a fluid-filled-saturated porous solid - ii. higher frequency range, Journal of the Acoustical Society of America 28 (1956) 179-191.

[9] M. Biot, D. Willis, The elastic coefficients of the theory of consolidation, Journal of Applied Mechanics 24 (1957) 179-191.

[10] M. Biot, Acoustics, elasticity, and thermodynamics of porous media, Twenty one papers by M. A. Biot, New York, Acoustical Society of America, 1992.

[11] J. Allard, Propagation of sound in porous media, Modeling sound absorbing materials, New York and London, 1993. 


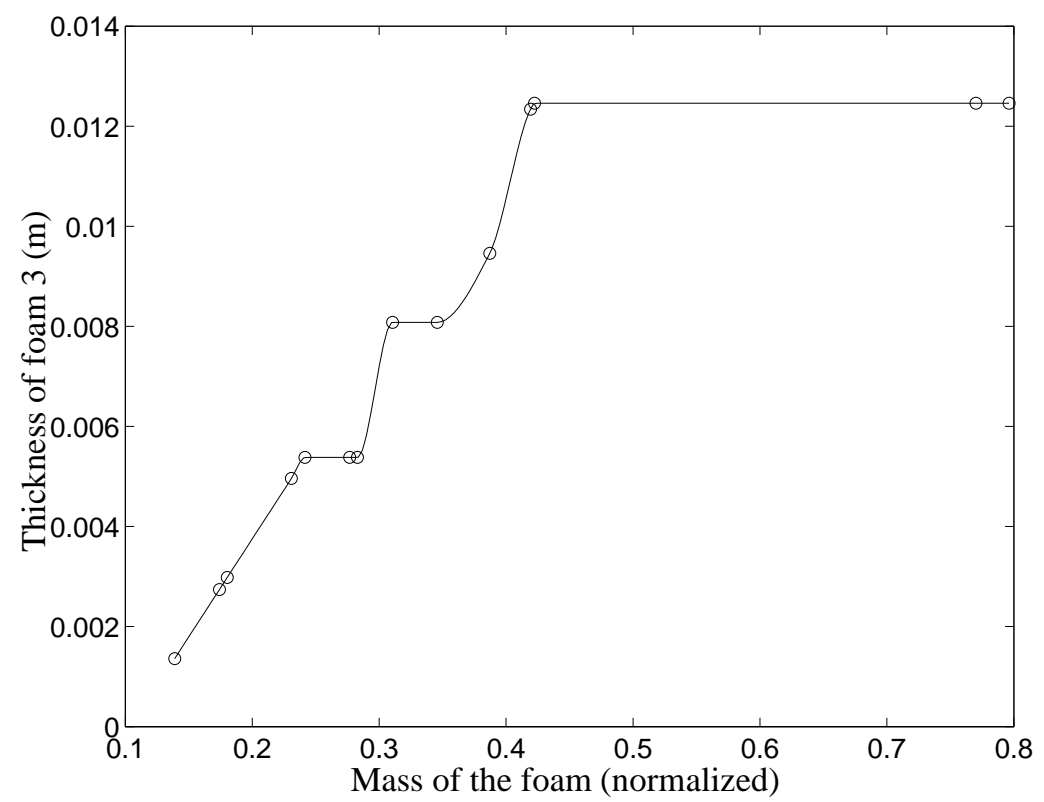

Figure 11: Best thickness of foam 3 in function of the mass of the foam

[12] Y. Champoux, J. Allard, Dynamic tortuosity and bulk modulus in air-satured porous media, Journal of Applied Physics 70 (1991) 1975-1979.

[13] N. Atalla, R. Panneton, P. Debergue, A mixed displacement-pressure formulation for poroelastic materials, Journal of the Acoustical Society of America 104 (3) (1998) 1444-1452.

[14] B. Faverjon, C. Soize, Equivalent acoustic impedance model. prt 1: experiments and semi-physical model, J. Sound Vibration 276 (2003) 571-592.

[15] B. Faverjon, C. Soize, Equivalent acoustic impedance model. prt 2: analytical approximation, J. Sound Vibration 276 (2003) 593-613. 


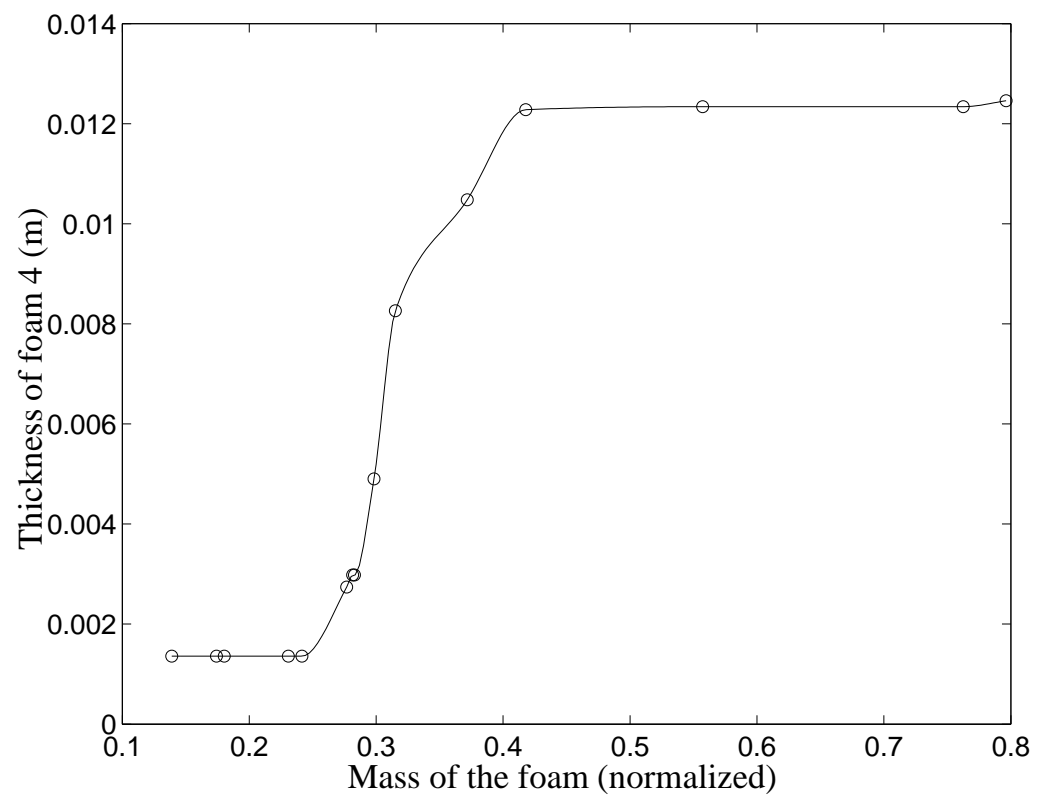

Figure 12: Best thickness of foam 4 in function of the mass of the foam 


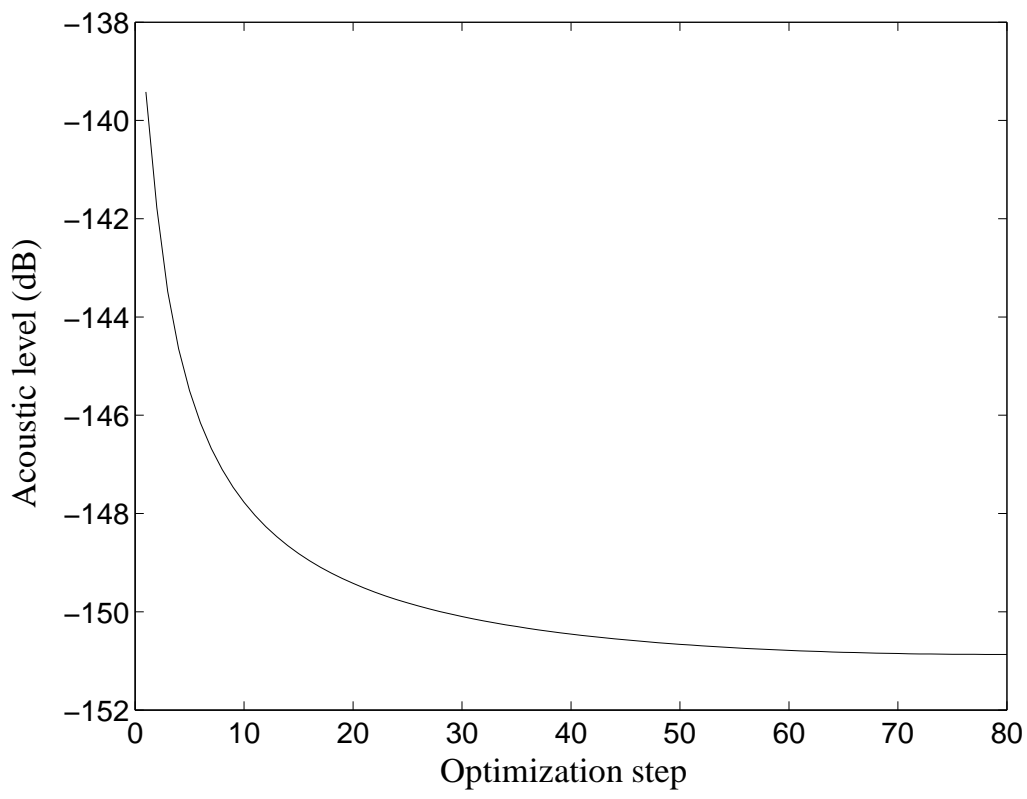

Figure 13: Pressure level in the first cavity $(170 \mathrm{~Hz})$ 